MODELING, IDENTIFICATION AND CONTROL, 1997, VOL. 18, NO. 2, 155-175

doi:10.4173/mic.1997.25

\title{
Model-Predictive Control and Real-Time Optimization of a Cat Cracker Unit
}

\author{
STIG STRAND* and LARS HÅKON VELAND**
}

Keywords: Model predictive control, on-line optimization, catalytic cracking.

A project for control and optimization of the Residual Catalytic Cracking Process at the Mongstad refinery is near completion. Four model-predictive control applications have been successfully implemented, using the IDCOM control software from Setpoint Inc. The most attractive feature of the controller is the well-defined control prioritizing hierarchy, and the linear impulse-response models have proved to give satisfactory performance on this process. Excitation and identification of the dynamic models proved to be a difficult task, and careful design and monitoring of the tests was mandatory in order to produce good results. Multi-variable Pseudo Random Binary Test Sequences were used for the excitation. Technical performance and operator acceptance of the new control functions have been good, but it is realized that a continuing effort is needed to fine-tune and maintain such functions.

An on-line optimization function based on a static yield and quality model combined with an economic model is currently under work. An extensive program of planned experiments, with careful logging of measurements and laboratory results is now completed, and a "hybrid" model established by a combination of theoretical knowledge and regression techniques fits the process responses surprisingly well. The chosen model complexity seems to be very cost-effective for this purpose.

The optimization is only local to the cracker plant. Prices for feeds and products have to be in accordance with the over-all refinery objectives to contribute to a global optimum. The new functions automate and integrate work and information transfer that were previously handled by procedures. This fact represents new demands for co-ordination and co-operation in the organization if the potentials of the new functions are to be fully exploited.

\section{Introduction}

The Residual Catalytic Cracking (RCC) process at the Mongstad refinery was commissioned in 1989, as part of a major plant expansion. Control structures for the reactor/regenerator at that time were fairly conventional, but they proved to be sufficient to stabilize the operation. Still, lacking constraint handling ability made it difficult to handle abnormal situations. Some more "advanced" features were implemented for the main fractionator and gas plant sections.

A long-term "Master Plan" for control and optimization prepared in 1992 by an external consultant recommended supervisory control of the RCC unit as the most beneficial of several projects. An internal engineering study recommended modelpredictive control as the preferred technology, and the use of external resources to do

Received 6 August 1996.

An early version of this paper was presented at the SIMS '96 Applied Modelling and Simulation Conference, Trondheim, Norway, June 11-13 1996.

*Statoil Research Centre, Postuttak, 7005 Trondheim (e-mail: stra@fou.statoil.no).

**Statoil Mongstad, 5154 Mongstad. 
the detailed design, programming, and commissioning, as this was the first project in this field.

The economic justification of the control project was based on two components:

- The ability to push the process closer to the prevailing physical constraints, because most of the time there is economic incentive to increase throughput.

- Reduced variability in product qualities, making it possible to maximize the yields of the most valuable products, within the physical and product quality constraints.

Expected pay-back time for the control project was 1 year.

An external consultant company (Setpoint Inc.) was responsible for the design and implementation of: a real-time database system, interface to the basic control system, MPC controller software, and MPC control applications. Additional expert assistance was hired from the Statoil Research Centre. A new VaxStation process computer was included in the project cost. All work related to the Bailey DCS system and field instrumentation was done by the refinery staff. This paper gives a control project review, offering some technical details along with project and organizatorial experiences. The four MPC applications have a total of 49 controlled variables, 33 manipulated variables and 13 measured or calculated disturbance variables. The reactor/regenerator and the main fractionator applications are focused here.

The Master Plan recommended an on-line economical optimization function on top of the MPC controllers, and this was also included as an option in the bids from the various vendors. At that time a "rigorous", kinetics-based steady-state model was foreseen, and the expected price was high. The optimization part of the project was however postponed because of budget reasons, and also because it was believed that familiarization with the controller part was necessary before optimization would make sense.

With the control project well done, we had formed the basis for the extension towards on-line optimization utilizing models for product yield and quality combined with economy and process constraint models to determine the sub-optimal Cracker operation. The insight in the RCC process resulting from the earlier theoretical studies (Ljungquist, 1990 and Strand, 1991) combined with the experiences from the implementation and commissioning of Model-Predictive Controls now made it possible to propose a simpler form of model and optimization function, with a much lower price tag. This project is going on just now with Statoil-internal resources only, and the project status is presented towards the end of this paper.

\section{Cracker Plant Overview}

The RCC takes oil feed from different sources, in varying mixing ratios, with the overall objective to upgrade the heavier parts of crude distillation to valuable product blend components. Most of the feed is usually atmospheric crude distillation bottoms (residue), either from our own crude unit or imported. Sometimes it is also incentive in upgrading through the RCC unit lighter stuff like the crude heavy gas oil, which is then mixed into the feed up to a certain ratio. The intermediate products from the RCC are:

- Dry gas entering the fuel gas system.

- LPG (liquefied petroleum gases, C3/C4 paraffins and olefins), which is further separated downstream to take out propene to be sold to petrochemicals, propane 
product and butane blend component, while the remaining olefins are reacted to poly-gasoline to enter the gasoline blending pool.

- Naphtha, to be used in the internal gasoline blending pool or sold as blend component to other refineries.

- LCO (light cycle oil) to enter the diesel pool after hydrogenation, or to be sold to other refineries.

- DCO (decant oil) which is further upgraded through the coker unit.

As shown in Fig. 1, the oil feed is brought to the riser bottoms, meeting hot regenerated catalyst circulating from the regenerator. The endothermic cracking reaction lasts for a couple of seconds in the riser, giving a range of hydrocarbons from dry gas to decant oil, which is separated from the catalyst in the reactor cyclones. The reactor effluent is directed towards the Main Fractionator, while the catalyst is circulated back to the regenerator to remove the deposited coke by combustion, stimulated by fresh air injection. The coke combustion process raises the catalyst temperature approximately 200 degrees, providing the needed heat for the riser cracking reaction. The regenerator combustion process has two stages, with intermediate catalyst cooling. This is necessary to prevent too high regenerator temperatures, while at the same time keeping a satisfactory level of catalyst activity. Coke deactivates the catalyst in a reversible way, while high temperatures cause irreversible hydro-thermical catalyst deactivation. The need for a two-stage regenerator comes from the residual feed, which gives a coke yield around 7-8 wt\%. More traditional catalytic crackers take feed in the gas oil range, and manage with a single-stage regenerator. Luckily, the intermediate cooling adds a very important degree of freedom when it is unconstrained, to control the regenerated catalyst activity and temperature independently.

The cracked reactor effluent is fed to the main fractionator, which separates the stream in overhead vapour, heavy naphtha, LCO and DCO. The overhead vapour is then fed to the gas processing plant and separated to dry gas, $L P G$ and light naphtha $\left(C_{5}\right.$ to

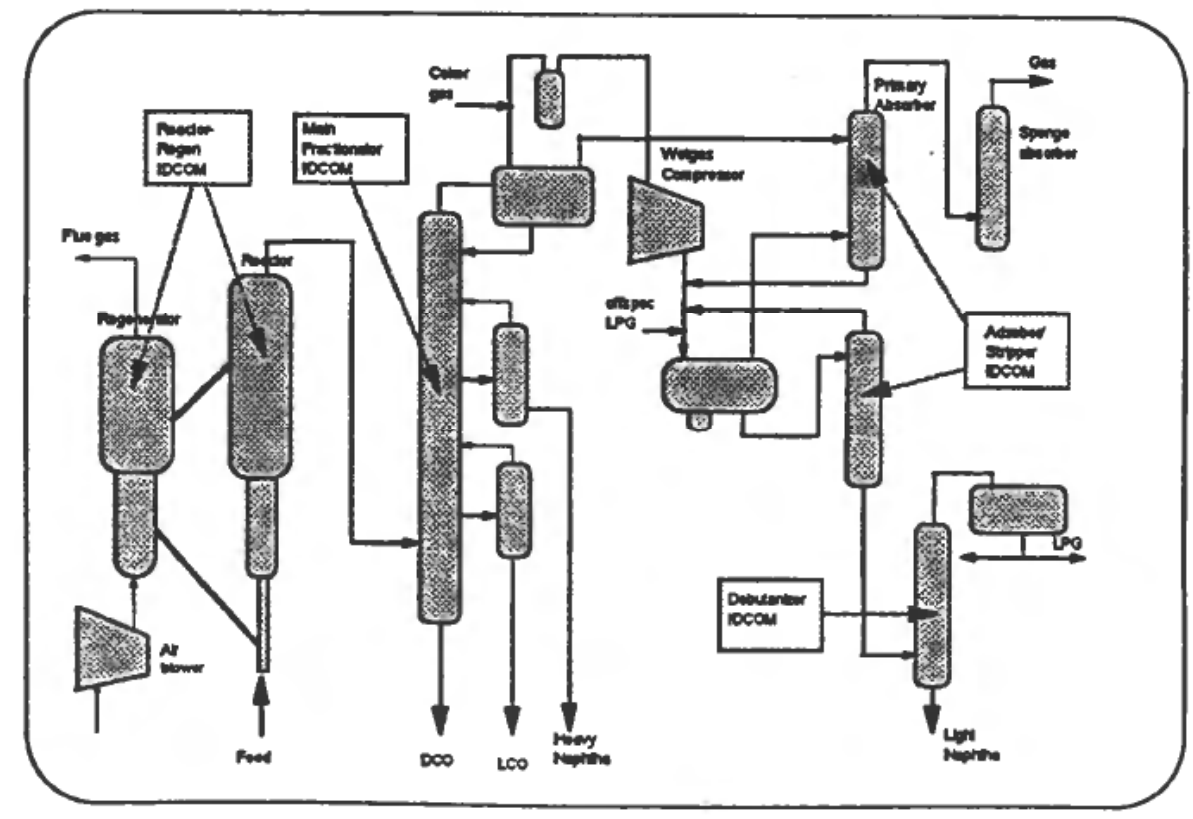

Figure 1. Cracker Plant Overview. 
a boiling point of approximately $170^{\circ} \mathrm{C}$ ). The light naphtha is then mixed with the heavy naphtha main fractionator draw-off to form the RCC naphtha product. A surplus of energy is brought to the main fractionator by the reactor effluent, which means that the circulation heat exchangers and steam generators remove heat from the column.

In addition to the riser oil feed, the RCC plant is fed with gas from the coker unit, entering the gas processing part of the plant just ahead of the wet gas compressor. The separated coker gas adds to the dry gas, LPG and light naphtha due to the riser feed.

\section{MPC Applications}

\subsection{The Controller}

The IDCOM controller offered by Setpoint was applied for all MPC applications. It can be briefly characterized by:

- Linear and constant single-input single-output (SISO) impulse response prediction models, superposing the models from each input variable (manipulated or measured disturbance) to the output variables, giving effectively a set of multiple-input single-output models which is considered in the control solution.

- Observed process and model discrepancy is assumed to arise from output step disturbances, and the subsequent predictions are corrected according to this assumption. Although this is a rather simple disturbance modelling, it does provide the attractive offset-free control when enough control freedom is available.

- An explicit control hierarchy is defined, going down from bounds and rate-of-change limits on manipulated variables via controlled variable hard constraints, soft constraints and set-points down to ideal values for the manipulated variables to approach whenever there is freedom left after satisfying the higher-prioritized objectives.

- A single-move restriction over the control horizon for each manipulated variable.

The first two points are quite common for commercially available MPC products, while the last two are specific for this controller. Our experience is that the control hierarchy is attractive, and we assume this feature will still be available in the future controller merged from IDCOM and DMC. The single-move restricts the controller bandwidth and decoupling severely, and the merged controller will not have this restriction.

Much can be and have been said about the SISO model representation, for instance that the resulting "complete" model order is definitely non-minimal since the basic process dynamics is "repeated" in most of the SISO models. From a practical point of view, the good thing is that model adjustments to improve the control can be done for each controlled variable separately, while the bad thing is that the basic process dynamics might show up slightly differently in the experimental SISO dynamics, which in fact may lead to undesired manipulation of input variables to reach control goals in dynamic conflict.

The vendor offered a Windows-based tool for controller configuration and simulation, which was very useful. The resulting configuration file was down-loaded to the Vax computer and the on-line application could be started straight off, with a gradual extension of the control problem handed over to the MPC. 


\subsection{Modelling}

Up to the time of our commissioning, the vendor had applied a sequence of step changes to each input variable of an application in turn, to collect the responses of all output variables to steady state. This is a time-consuming procedure, requiring a stable process operation with "all" other input variables left constant, at least in order to determine the effects of the input variables picked out first. To come around this, he proposed running a pseudo-random binary sequence (PRBS) step signal on several inputs simultaneously, an idea which was adopted for the project. Typically, two to four inputs were grouped together, for which a PRBS was run over a 10 to 14 hours period. The operator was left with the possibility to change the average level of the test signals for each individual input to "control" the process, but operations was also heavily consulted in determining the step sizes. Naturally, the operator also had the option of turning the PRBS off, but this was never done even during nights when no engineers were available at the plant.

At first glance, this method was attractive in the sense that a lot of testing time was saved. The models were identified by a combination of high order MISO ARX and subsequent model reduction, before finally forming the SISO impulse responses needed for the controller for the models having a satisfactory degree of estimated certainty. As part of this process, we had a lot of discussions on expected relationships based on process knowledge.

However, during the fine-tuning period after commissioning we detected several severe discrepancies in some important models, and repeated single-input testing gave us models which raised the control performance significantly. Looking back, part of the reason is that we were not clever enough in determining the frequency content of the PRBS, with a too high average frequency giving us reasonable initial dynamics good for a traditional regulatory controller, but less good models towards steady state, which is also very important for an MPC solving for feasible constraints at steady state. Another reason is probably that judging the process behaviour during testing is much more complicated when applying a multi-input PRBS, which led us to over-look some severe non-linear effects like that of the wet gas compressor valve illustrated in the next section. To conclude, we still believe in the PRBS-type of testing, relying on the fact that this first experience improves our own performance for the coming projects.

\subsection{Reactor/Regenerator MPC}

\section{Objectives}

The main objective of the MPC is to stabilize control and to keep the process against a varying set of active constraints, arising from time-varying economic objectives from the planning department like maximization of throughput or conversion (percentage of products lighter than $215^{\circ} \mathrm{C}$ boiling point). The controlled variables, mostly constraints, are related to:

- catalyst slide valve positions and delta pressures (catalyst circulation)

- catalyst cooler steam production

- $\mathrm{CO} / \mathrm{CO} 2$ ratio of the regenerator flue gas (combustion control)

- regenerator temperatures (metallurgical and irreversible deactivation)

- regenerator air blower loading

- wet gas compressor loading

- downstream processing capacity

- main fractionator loading 
while the important manipulated variables are:

- feed rate DCS setpoint

- reactor temperature setpoint to DCS controller which manipulates regenerated catalyst slide valve position

- second stage regenerator temperature setpoint to DCS controller manipulating the catalyst cooler slide valve position

- air rates to first and second stage regenerator, respectively

- reactor/regenerator delta pressure

- wet gas compressor suction pressure

- gas rate from the coker unit

There is a total of 11 manipulated and 26 controlled variables in the application, which has attained an average service factor close to $98 \%$ over the last year.

The MPC application does not include yield and quality models for the hydrocarbon products. They are focused in the optimization project referred later in this paper, which will produce ideal settings of the important cracking handles like feed flow, composition and preheat, together with reactor and regenerated catalyst temperatures.

\section{Modelling Issues}

The regenerator flue gas $\mathrm{CO} / \mathrm{CO} 2$ ratio is controlled to maintain an appropriate combustion level. The on-line analyzer is routinely taken out for service. In order to leave the MPC on and still have reasonable combustion control we developed an estimator based on raw measurements available from the DCS. The estimator follows the analyzer dynamically, but tends to drift with time. However, we update a bias correction factor for the estimator as long as the analyzer is good, and are then in a position to use the estimated value for control also when the analyzer drops out. This is an example of a relatively simple technique for a control engineer, though necessary to keep up the service factors of a complicated multivariable control application.

As the dynamic models in the controller are linear and constant, it is very important to apply a model identification testing sequence which gives rich information in the interesting operating range of a controlled constraint variable. The wet gas compressor steam valve is a typical active constraint on the high side, with the actual constraint limit typically around $95 \%$ above which additional opening has almost no effect. The MPC input variables with major effect on compressor loading are:

- riser oil feed rate and temperature

- reactor temperature

- compressor suction drum pressure

- coker gas

Actually, all variables affecting the cracking do have compressor loading impact, but those mentioned above explain the most.

The 235 data points shown in Figs. 2 through 4 come from the data set collected as part of the optimization project, and here we have used the one-hour averages for the valve opening and all the input variables. This should give a good approximation to a stationary model, as the time constants of the dynamic valve response are a few minutes because the light product parts of a riser oil feed or reactor temperature increase will show up very rapidly as main fractionator overhead vapour and the hydraulics of the overhead vessels is fast. 


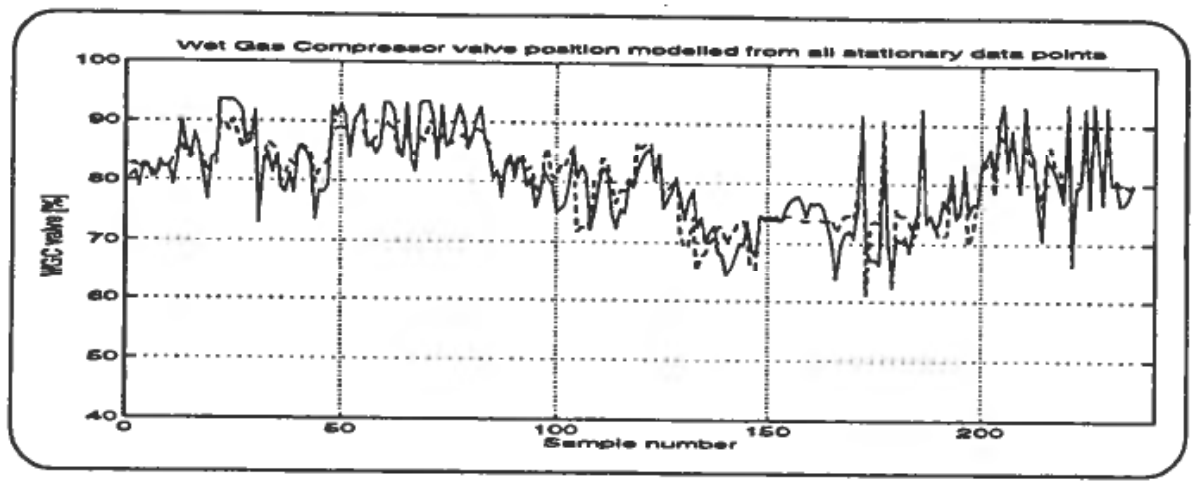

Figure 2. Wet Gas Compressor valve position, modelled from all stationary data points.

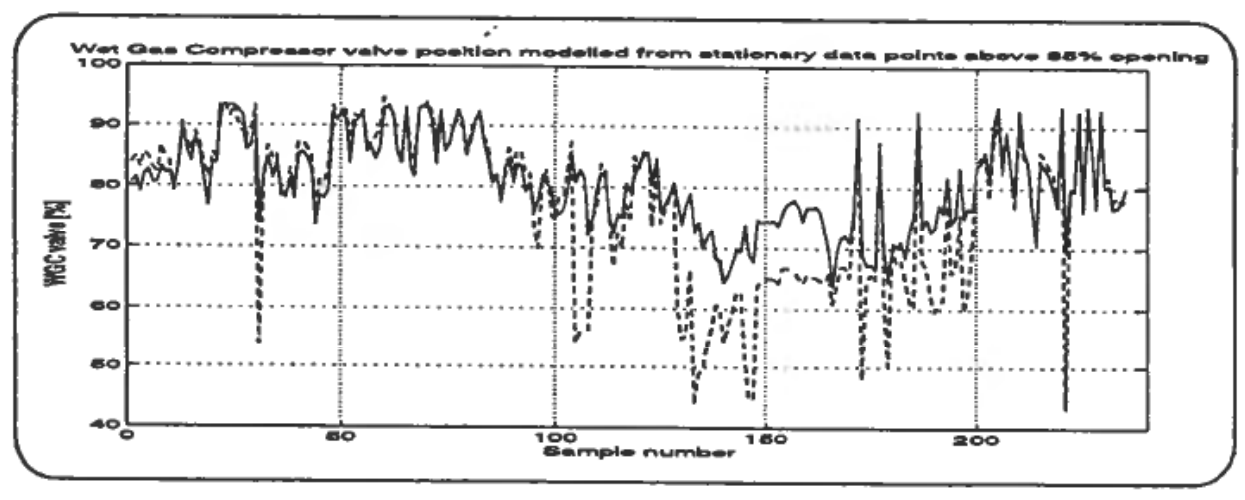

Figure 3. Wet Gas Compressor valve position, modelled for valve openings above $85 \%$.

Figure 2 shows the measured valve position (solid line) and the linear model response (dashed line) established from all available data points, while Fig. 3 illustrates the effect of including only measured valve openings above $85 \%$ in the modelling. Clearly, the fit in the interesting range is much better in the latter case, with model coefficients about twice the values of the former. There are two causes: the valve has a non-linear characteristic, which means that a certain load change gives much more delta valve opening in the $85-100 \%$ range than below $80 \%$; and the operators increase the compressor recirculation flow to avoid surge at lower loads. Including the recirculation as a known input is fairly easy, but unfortunately we did not have it in the data set. The valve nonlinearity is not that easy to come around with the linear model assumption, but is handled well by the combination of modelling in the correct operating range and a reasonable MPC constraint limit specification. As part of the optimization project we might include the recirculation as an optimization variable, in order to let the optimizer see the real room for overhead gases.

Figure 4 shows the stationary part (gains) of the MPC models. Firstly, we see that the modelling based on the dynamic testing through the commissioning, which was more than a year before we started collecting the shown data set, was done in the correct range. This should always be assured during MPC commissioning. Secondly, we should add that we did not perform a satisfactory pre-testing against that valve before the PRBS was applied. This gave us PRBS valve responses with much data well above 


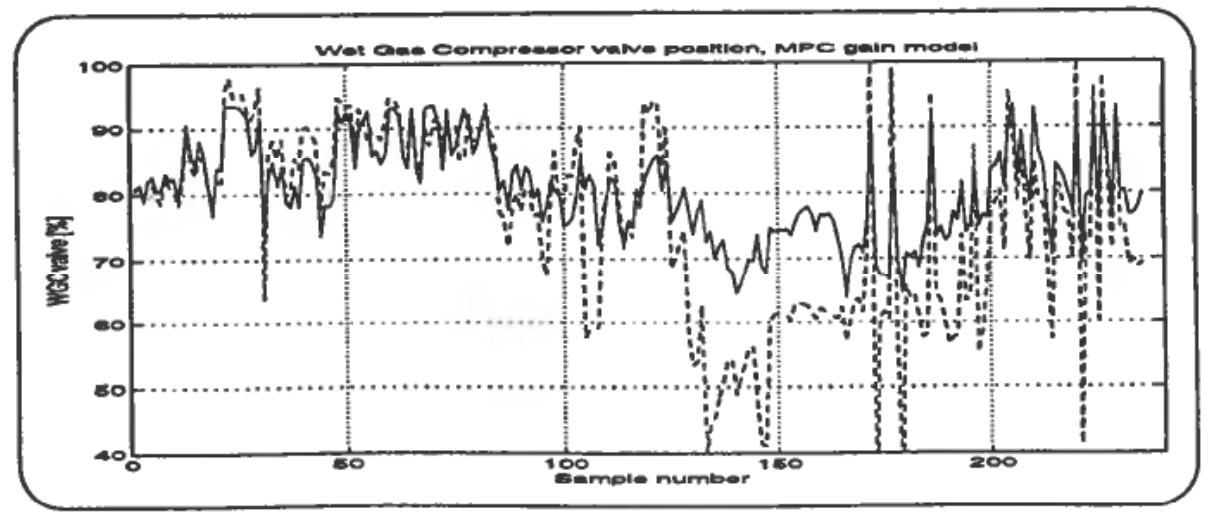

Figure 4. Wet Gas Compressor valve position, MPC gain model.

95\% opening, where additional opening has minor effect, leading to large overshoot models in the controller. During the MPC fine-tuning period we realized what had happened and rebuilt the models with a major performance improvement.

Finally, this example should also initiate an interesting discussion on the dynamic model identification issue. We think that a lot of effort could have been saved during commissioning if steady-state model coefficients were available in advance, based either on an informative "stationary" process data set or on a verified rigorous process simulator. The full utilization of such information calls for a dynamic model identification tool accepting fixed steady-state gains as input data. Highlighting only the dynamics in the PRBS testing would save a lot of testing time. Compared to a more traditional high-bandwidth regulatory controller, a model-predictive controller needs to predict stationary responses fairly well to reach and stay at the correct active constraint set.

\section{Where is Optimum?}

All variables influencing the compressor loading also affect the product yields and qualities from the Cracker plant. As there are no such models in the MPC, we implemented a prioritizing structure with handles to change the sequence of backing off feed rate, coker gas or reactor temperature to alleviate a compressor loading constraint violation. IDCOM makes this possible by the attractive control hierarchy allowing to prioritize explicitly between manipulated variables whenever there are degrees of control freedom left after satisfying all specifications for the controlled variables. However, this sequence switch handle reflects by no means the optimal adjustment of the manipulated variables in a compressor constraint situation, which depends on a combination of product and feed economics, yield and quality effects and the complete set of active plant constraints. This illustrates the need for an on-line optimizer on top of the MPC system to determine the optimal adjustment.

\subsection{Main Fractionator MPC}

\section{Objectives}

The main control objectives are:

- stable control of the fractionator top and bottom sections (temperatures and levels) 
- product quality control under valuable product maximization

- meet operating objectives in the bottom and product-draw sections

The main fractionator MPC has 15 manipulated variables and 8 measured disturbances, affecting the total of 13 controlled variables, with the heavy naphtha $95 \%$ boiling point as an important one in the sense that the constraint is nearly always active. This MPC application is by far the most challenging one, supporting the common opinion that cracker main fractionators are difficult. Contributors to this fact are slurry pumparound coking and carry-over catalyst problems, affecting the pumps and heat exchangers severely. Also the high level of heat integration both with riser feed preheating and downstream distillation columns makes main fractionator operation more cumbersome.

A well-educated control engineer would probably think that this truly multivariable process should be controlled by an MPC knowing and exploiting all the process interaction. But tell the operator that he should enjoy the top reflux being used to control the bottoms temperature below the high limit and see how he reacts. So we had to use dirty tricks like duplicating the top reflux in the MPC, as a manipulated variable to control the upper part and a measured disturbance against the lower part controlled variables, the latter with reduced gains compared to that of the identified models. In some cases it is clear that the single-move restriction of IDCOM rectified such problems, limiting the attainable dynamic decoupling.

The main fractionator MPC application has attained a service factor close to $90 \%$. We think this is a reasonable figure, because the remaining $10 \%$ represents process upset periods of the kinds mentioned above which are handled much better by the operators than by a multivariable MPC with fixed models.

\section{Modelling Issues}

The naphtha product final boiling point (RCCN FBP) is controlled due to specifications on the blended gasoline. Very often, there is a large incentive in maximizing the naphtha flow up to the FBP specification. The naphtha FBP is implicitly controlled by the heavy naphtha $95 \%$ boiling point (HN95) from the main fractionator, as they correlate very well. An estimator has been established for the HN95 based on several process and laboratory data points. The estimated HN95 is compared to the laboratory distillation value for several data points in Fig. 5.

The HN95 estimator is a regressed combination of raw measurements like distillation tray temperatures and calculated variables like heavy naphtha tray $\mathrm{L} / \mathrm{V}$ from mass- and energy balances. The model is a linear regression of 10 variables. The correlation fits almost equally well when only raw measurements are used. Figure 5 shows a time period with bad fit between sample 150 and 200. This period has not been included when establishing the shown model, due to severe process problems, and the discrepancy cannot be explained by any combination of available regression variables. The overall model error standard deviation for the other samples is 2.3 degrees, which is close to the laboratory accuracy. Using the estimated HN95 as a controlled variable in the Main Fractionator MPC has reduced the Naphtha product FBP variance, with a resulting potential of raising the average value approximately 2.5 degrees. The benefit is an increased naphtha yield in RCC naphtha priority periods.

We started collecting the data systematically in parallel with the commissioning period. As the data grew, we observed periods where the "best" available estimator at that point in time drifted away from the laboratory result. The estimator was then 


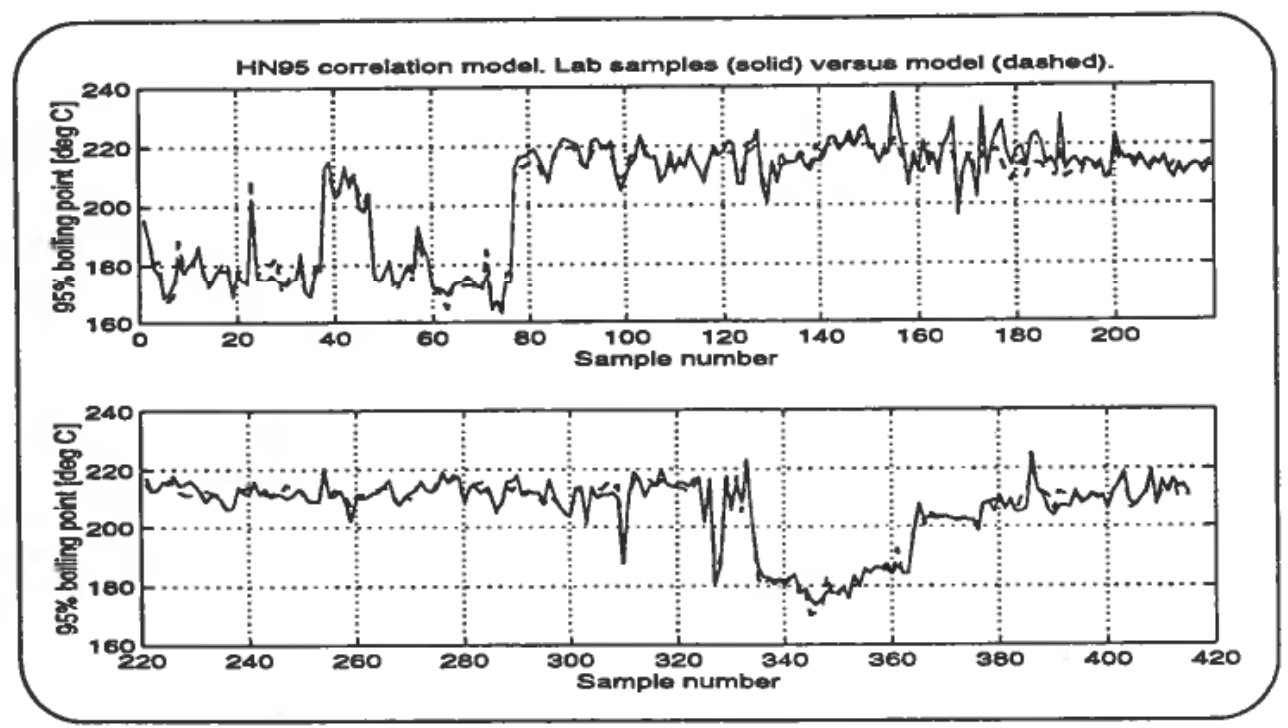

Figure 5. Heavy Naphtha $95 \%$ boiling point correlation.

re-calibrated based on the entire data set, and with the 415 points currently available with a lot of process variability we believe that there is little additional information to pick up in the future. The lesson learned is that systematic data collection should be started well ahead of such a control project, and that most refinery processes would benefit from quality estimators in lack of on-line analyzers, regardless of the implemented control level.

\section{Project Experiences}

The design and programming phase went essentially according to schedule. Substantial modifications of the basic control functions in the DCS system were necessary to prepare for computer control and to remove the control structures made obsolete by the IDCOM controllers. These modifications could not be done under normal operations, a planned maintenance stop the autumn 93 was the only opportunity. Commissioning of the supervisory control could start when the plant was back in normal production.

The commissioning period was severely disrupted due to process problems not related to control. Many special conditions had to be fulfilled to run the PRBS test sequences, and field instrument problems were common. Based on that experience, we advise others doing project of this kind to carefully identify and solve the instrumentation problems in advance of commissioning, as this will save a lot of time and effort. However, the defined project (Setpoint delivery) was finished at estimated cost, but several months delayed. At this stage all controllers were operational, but not tuned to maximum performance.

\section{Evaluation}

The identified pre-conditions for obtaining the economic results were:

- Formal limitation in throughput to be increased by $15 \mathrm{~T} / \mathrm{h}$.

- Removal of capacity constraints in downstream units. 
- Tuning and modification of control structures for downstream units, to handle continuously varying feed flow.

A special evaluation was made after one year of operation. Technical performance and pay-back due to reduced variation and better constraint control was as expected, but it should be noted that this performance was reached gradually after a substantial tuning and monitoring effort which lasted almost a year after the nominal start-up of the multivariable controllers.

The economic benefits from pushing throughput was less than expected, this was not due to control performance, but because a formal limit on throughput related to flare system capacity had not been relieved at the expected time, and because downstream processing capacity had not been increased.

\section{Operational Limits and MPC}

Well-accepted operational limits related to physical equipment have been implemented as constraint-controlled variables in the MPC. This represents a two-edged sword. In contradiction to an operator adjusting basic control setpoints manually, the controller respects the limit from minute to minute, and does not accept "dynamic" violations for a couple of hours. If we disregard the long-term economical impact of violating such limits, which can be very hard to evaluate and related to equipment tearing, there is a possibility that MPC manipulated variables which are important for the short-term and identifiable plant economics take average values which reduce that part of the economics. Typically, this might cause problems in evaluating the economical benefits of an advanced control project. The lesson is probably that if a performance evaluation is to be done after the project, the value of respecting operational limits and the performance in doing so without advanced control should be determined in the early project phase. However, a control engineer, but probably not a process engineer, would say that "everyone knows" that more stable and consistent control is better for the plant.

\section{Lessons Learned}

The IDCOM MPC software performed well for our applications, after a couple of serious bugs were detected and corrected. The main advantage is the constraint handling performance, and the flexible and user-friendly control priority hierarchy.

A significant effort was required to bring the performance of the MPC controllers from an acceptable state to best attainable. A working, stable controller is just the beginning. Continuous observation, analysis, and modification is needed. This joint effort by the Statoil Research Centre and the refinery staff took us a year or so. Looking back, we believe it was best to do this by Statoil-internal resources instead of using the external consultant. Interactions between the process and the control functions are complicated and takes time to learn, and this very valuable experience should remain in the plant, not in the consulting company.

Identification of the dynamic models is a critical issue. The dynamic models in IDCOM are linear, and testing for model identification must take place in a linear operation regime.

The limited precision in control valve positioning due to friction/hysteresis can limit the achievable performance of supervisory control functions. The problem is not easy or cheap to fix, and this very practical problem seems not to get the attention it deserves when control algorithms are designed nor when their applications are specified. 


\section{Critical Success Factors}

At the start of the project the following criteria was emphasized:

- Active interest and commitment from the users, i.e. operations department managers and panel operators.

- Monitoring of utilization after commissioning, Keep information updated.

- Keep up know-how by continuous activity in the field.

In many ways, supervisory control functions fall between the domains of the traditional automation or process control groups and the process/operations engineer groups in a plant. In our case ownership was definitely in the process control domain. In addition, one experienced operator was appointed as a full time member of the project team. He has been a key person in most of the development and implementation activities.

MPC technology is significantly more complex than conventional controls, but, surprisingly enough, this has caused little resistance to the controls by the end users, i.e. the panel operators. A one day demonstration/training session on our training simulator system, and a long commissioning period helped to ease the introduction.

The new controllers very soon proved to be safe, and the various protective mechanisms proved to work, and when functions are trusted, and makes life easier for the operator, they are used. It has been more of a problem that controllers have not been disconnected when the prerequisites for their use are not present, than reluctance to activate them. Complaints now, after more than 2 years of operation, is that the lack of "excitement" makes it difficult to handle abnormal operation. Simulator training is the obvious cure for this, but that is another story.

Participation in the implementation and commissioning phases is extremely important in order to learn the interactions between the process and the multivariable controllers, and thus to acquire the "ownership" of the new functions. In our case the process engineers were less involved than the process control and the operations people in this phase of the project, and we now realize that it is difficult to transfer the experience and know-how to other persons at a later date. The result is that there still is some uncertainty about the organizational responsibility for the utilization of the supervisory controls. The whole refinery organization is currently under revision, the outcome is uncertain at this writing, but a clear allocation of resources and competence is necessary on order to ensure monitoring and maintenance of the control and optimization functions in the future.

The ownership problem may be related to the engineer education in Norway, where systems engineering and dynamics are more related to software, theoretical cybernetics, and mechanical curriculums. Process engineers usually have a chemical/process design background, with less emphasis on dynamics.

Our experience is that the effort required to maintain supervisory controllers should not be under-estimated. There are lots of numbers that have to be continuously evaluated and adjusted when the process itself, the production objectives, or the control objectives change, as they do.

The operator interface for our MPC controllers leaves much to be desired. None of the vendors in question offered much in this respect. Here is definitely room for much improvement, given the graphics capabilities of modern control systems. 


\section{Optimization}

\subsection{Background}

The optimal operating point of the Cracker plant depends on a large diversity of conditions like:

- Product prices and direct volume and quality specifications at the downstream edge of the Cracker, reflecting the further utilization of the (intermediate) products. For the products going straight to export the actual market prices and contracted deliverables will influence, but for those entering a blending pool one has to consider the current pool situation with respect to component volumes and qualities, the expected component deliveries from other plants in the refinery and finally the scheduled blended-product deliveries.

- Feed prices, which reflects both alternative utilization and the availability of feed components.

- The reactor/regenerator cracking conditions, which depend strongly on feed rate and composition, reactor temperature, and catalyst formulation, circulation and activity.

- The Main Fractionator operation (distillation, heavy naphtha/LCO cut-point, operating objectives not related to product economics) and the distillation in the gas processing unit.

- The refinery energy situation.

- Cracker-internal and downstream processing constraints due to equipment limitations.

Traditionally, just a few of the available Cracker handles influencing the economy are actively used, the rest stay more or less constant at safe average values. The obvious reason is that the interactive responses make it difficult for a human being without a formal process and economics model at hand to predict the consequences of simultaneous changes. Realizing this led us to pick up the previous idea of an Optimizer on top of the well-performing MPC system.

Obviously, the feed and product prices and specifications strongly influence the location of optimum. The scope of the current optimization project does not include the price-balancing mechanism which has to be developed to assure that the Cracker sub-optimization contributes positively to the global refinery profit, but does naturally include the implementation of a large flexibility in specifying Cracker edge-conditions.

During project planning, we identified modelling of the cracking in the reactor/regenerator and of the product consequences of Main Fractionator operation as the key process modelling activities to reach an optimization function which could give us substantial increase in the plant profit. There was already an available vendor model used by both production planners and process engineers at the refinery. However, we did not trust that model too much, because it had never been appropriately verified against our process and it is basically a regression model based on several data points from several refineries, with a large diversity in Cracker design, feedstocks and catalysts. Hence, a large plant testing plan was prepared with a two-fold objective: to verify the available model and to have enough data to build new models should the vendor model performance be unsatisfactory. The current state is that we have finished the testing period, rejected the vendor model for our purposes and built our own models which are much better for our specific Cracker.

Furthermore, we realized that the most economical operation point for the cracking 
process will most of the time be close to equipment and/or quality limits. This means that knowledge of the actual process operating point relative to the real limits is necessary in order to calculate a feasible and realistic optimum, and that the MPC control functions are necessary to provide safe and stable operation at the optimum. The optimization function was thereby planned to operate on line, continuously collecting process values and updating models accordingly.

Developing an optimizing function like this to be a tool for the process operator to some extent transfers responsibilities from production planning to process operations, and to some extent requires a change in the type of production plans directed from planning to operations. The consequence of this was that all affected parties had to take actively part in the project.

Although the project is not yet finished, we will provide some insight in the following, on modelling as well as organizatorial issues.

\subsection{Plant Testing}

\section{Experiment Design}

The experiment design focused on the most important cracking variables like reactor temperature and the temperature of regenerated catalyst, which together with the feed rate and temperature to a large extent determine the catalyst circulation and hence the catalyst to oil ratio. Perturbing these three temperatures and accepting the feed specified from the planning department or adjusted by operations against downstream processing constraints was believed to give a lot of cracking information. In addition, we included perturbations of some other variables. The perturbation sizes were agreed as a trade-off between the desire of spanning out a wide operating range and considerations done by process engineers and operations with respect to their process knowledge and earlier experiences.

There is a lot of natural variation in the Main Fractionator operation, stimulated both from the production planners and from necessary adjustments judged by operations. During the major test period there was a minor and separate Main Fractionator naphtha/LCO cut-point test carried out to re-calibrate the heavy naphtha $95 \%$ boiling point estimator. Hence, for the major plant test no specific Main Fractionator perturbation was specified since the data information content was expected to be rich anyway.

The time constants of the process from the reactor/regenerator to the product end, for the product parts going through the gas processing plant, are in the order of one hour. Hence, after a change in say the reactor temperature, the entire effect shows up after approximately 4 hours at the products. In addition, there are never-ending plant disturbances which cause variations in the product draws. We found that the best trade-off between data consistency and individual test duration was to implement the input change, wait for 4 hours and then collect data for the next 4 hours, before the laboratory samples were taken. Considering the time needed to implement the input change, this gave us a total of 10 hours duration for each individual test.

\section{Data Collection}

A spreadsheet tool was developed to collect data records. When the laboratory results were ready, say a day or a week after a certain test, we entered the lab data manually into the spreadsheet, and used the sample time stamp to go back in the INFOPLUS (the real-time database offered by Setpoint) history via DDE links to fetch 
appropriate process data for the four-hours period ahead of the time stamp. As we used 12-minute averages routinely aggregated by INFOPLUS the data was available for 35 days back in time. Based on the 12-minute averages, the spreadsheet macros calculated and stored 1-hour and 4-hour averages and standard deviations, offering us the possibility to determine the process variation level at the time of modelling. The spreadsheet tool has now been extended with an interface to the laboratory database so that no additional manual data entry is necessary for this purpose.

Deciding which process data to collect and which laboratory samples to ask for was a joint venture between representatives from operations, process control, technical process support and design, laboratory and production planning. We ended up with collecting approximately 250 process values (measurements, calculations, relevant MPC entries).

The testing period lasted from July last year until Easter this year, separated by an entire refinery maintenance stop last autumn. Based on the uncertainty level of the modelling performed on the first-period data set, we realized the need for approximately 30 new test runs after the shut-down. In addition to the total of 60 test runs we also collected data from normal operation, with only routinely laboratory data available. The normal operation has process variation as well, and the method of data collection allowed us to pick only good data for modelling.

Process testing of this kind naturally requires the process operation to be shifted from the normal mode, but our experience is that though the product yields and qualities changed, sometimes leading to reduced throughput, the perturbations were well accepted by the planners and operations and the complete testing programme went very well.

\subsection{Modelling}

\section{Naphtha Example}

The process models for the optimization should include all independent variables with a certain effect on the plant economy as well as Cracker-internal or downstream constraints. The RCC Naphtha product is used as a modelling illustration.

The measured naphtha product flow and quality depends on:

- the oil feed rate and composition

- riser/reactor cracking conditions

- the Main Fractionator operation (distillation, heavy naphtha/LCO cut-point)

- coker gas rate and composition to the gas processing unit (some light naphtha)

- a couple of reprocessing streams occasionally in there with varying composition

- a lot of plant vessel dynamics

- naphtha flow measurement errors, mainly due to varying density.

When modelling the naphtha product flow, we excluded 124 of the entire set of 235 points, based on a judgement of the standard deviation of important measurements within the 4-hours averaging period. The remaining 111 points are encircled in Fig. 6.

We realize that most of the points from the planned plant testing are still in there, represented mainly by points of high and low reactor temperature. This is natural due to much more restrictions on the plant operation throughout a testing day, and also because some of the tests were repeated when unacceptable plant conditions were observed.

Average composition of coker gas and reprocessing streams were calculated, and 


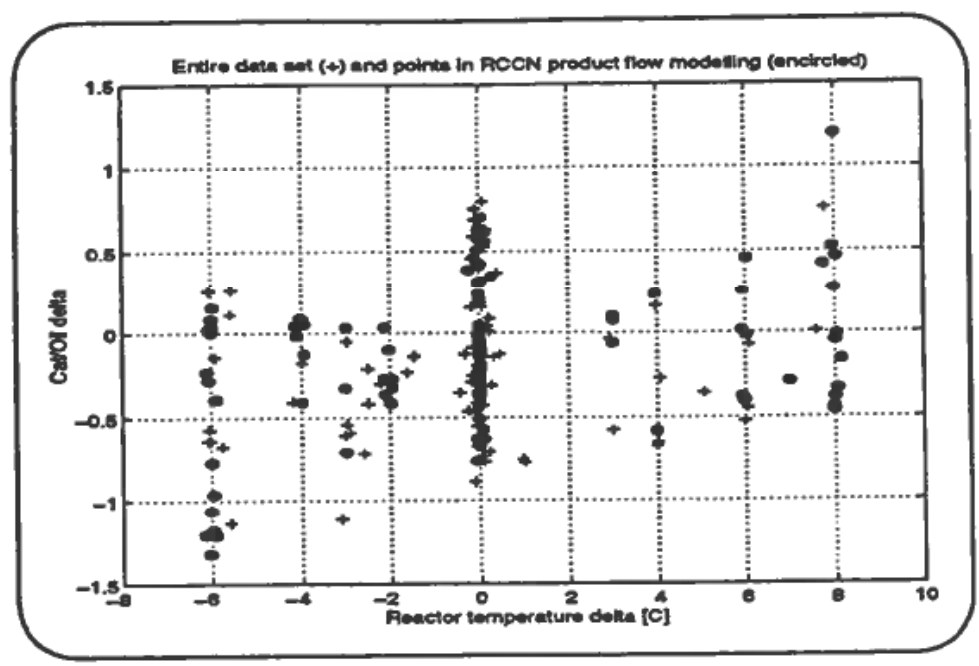

Figure 6. Cat/Oil and reactor temperature values in data set.

the predicted effect of those flows were subtracted from the naphtha product, which gave us a calculated naphtha product flow with the origin of oil feed to the RCC riser.

By these pre-cautions, we were still left with a noisy data set with each source too small to affect the model severely, but with the probability of adding up effects at certain points. The oil feed composition was analyzed for about half of the 111 points. However, the ratio of crude tower heavy gas oil to total feed rate which has a large impact on feed composition was known from measurements. During the modelling sequence it was concluded that detailed feed composition knowledge did not reduce the modelling error significantly, although different types of oil residue were fed during the test period. This conclusion is certainly invalid for crackers with much more feed variation. Hence, the model response shown in Fig. 7 is based on:

- the oil feed rate and ratio of heavy gas oil

- riser/reactor cracking conditions

- the Main Fractionator operation (distillation, heavy naphtha/LCO cut-point)

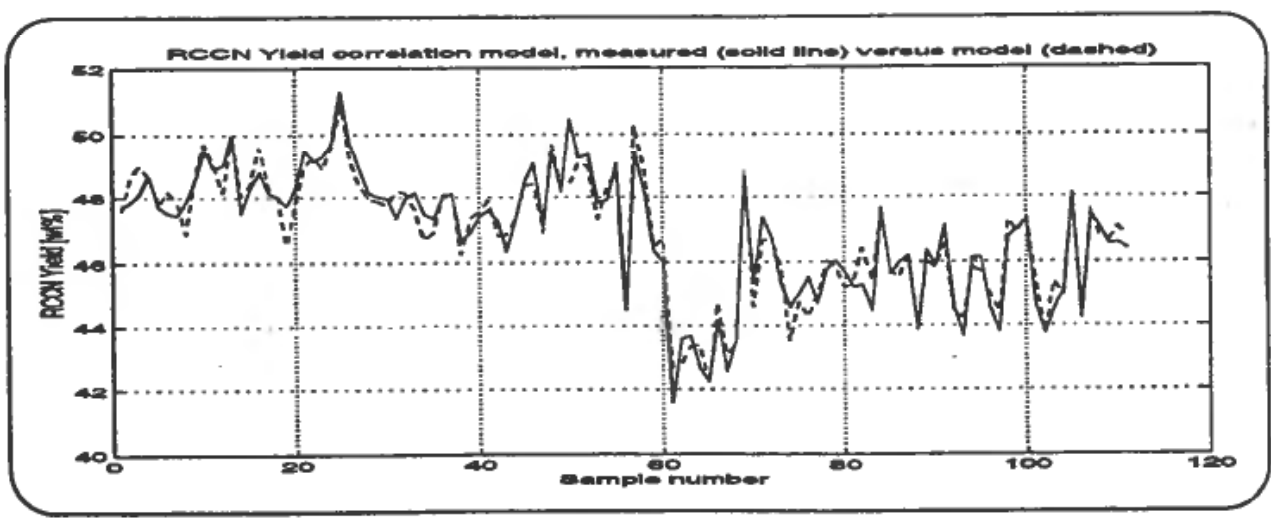

Figure 7. RCC Naphtha yield from riser oil feed. 


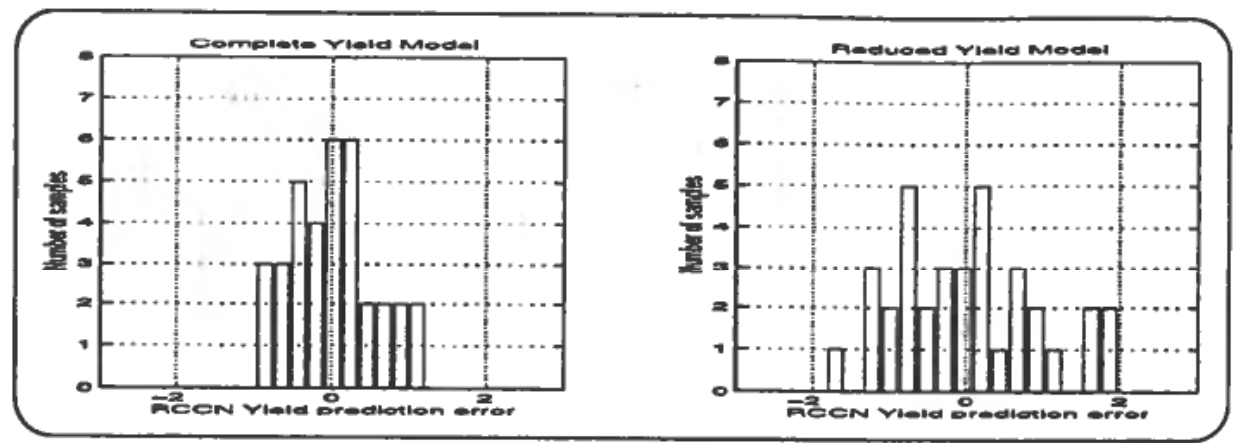

Figure 8. Effect on RCC Naphtha Yield prediction error from cracking variables.

The yield is shown instead of product flow to remove the direct effect of feed rate variations. The prediction error standard deviation is $0.6 \mathrm{wt} \%$, a result which we think is fairly good and close to the noise level. This is achieved with 9 correlation variables, some of them entering the model equation nonlinearly. Three of the variables come from the Main Fractionator, the others from the reactor/regenerator.

The effect of including cracking variables like reactor temperature and cat/oil, as opposed to modelling only from throughput and Main Fractionator operation, is illustrated in Fig. 8. The left histogram shows the prediction error distribution with the above model, where the accepted data points from controlled plant testing are included (i.e. those with most variation in cracking conditions). The right histogram shows the corresponding errors from a model established from throughput and distillation.

The value of RCC Naphtha depends also on the octane number. The RON and MON laboratory results were available for approximately 100 acceptable data points, as shown in Fig. 9 along with the model responses. The model errors are in the order of laboratory precision, and precautions to avoid over-fitting have been taken. So within the operating range spanned out in the data set we feel very confident also with the octane number predictions.

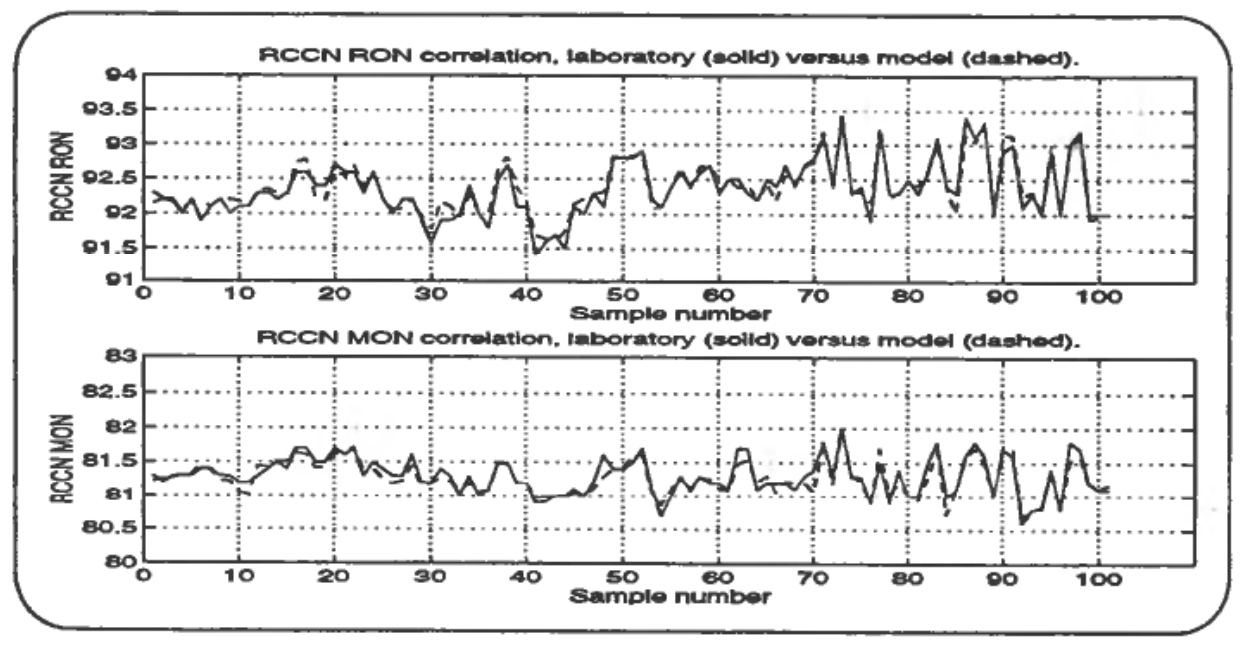

Figure 9. RCC Naphtha RON and MON correlation. 


\section{Modelling Discussion}

The models of the other important responses in the Cracker plant have an accuracy comparable to the naphtha models, and they seem to be good enough to start turning on-line optimization on. There are several reasons for the success of the modelling phase, some of which are mentioned in the following.

Process testing was planned and carried out carefully, perturbing the important variables as much as possible within the restrictions set from operations and planning. We also had a sound attitude to process dynamics and noise in advance, influencing the number of test runs and the method of data collection, which again made it possible to judge the process stability for each data point.

With the MPC controllers we were able to reach the specified operating points significantly faster and keep them in a more stable way for the needed time period than without them. That we know from earlier similar testing experience. And with the INFOPLUS system installed, we were able to fetch all the needed process values easily, and also do some on-line calculations of mass- and energy balances giving us both estimated coke production and catalyst circulation rate.

A lot of research has been done on Cracker yield and quality models (see Ljungquist, 1990 , for a review). We have to some extent looked into these publications and applied some of the ideas, basically in forming correlation variables. Our current conclusion is that rigorous cracking modelling is not worthwhile for our purposes. This is based on what we have achieved by simpler means compared to the process noise level, the expected complexity of the needed kinetics and the observation that "everyone" starting off with rigorous modelling of this process finally lumps and does need a high degree of data regression to fit the lumped parameters. And we think that procedure has to be repeated for instance when changing catalyst formulation, as it certainly will also in order to re-calibrate our models.

\subsection{Optimization}

\section{Methodology}

On-line real-time optimization is an "in between" function between the production planning and operations groups. Product prices are settled a week or so before actual production. At that time only expectation values are available for the state of the process. In actual operation, the yield and quality numbers produced by a nominal model will never exactly match the measured result.

Our solution to these problems is to make two versions, tailored to the needs of each user group. The two versions will have identical kernels, consisting of the basic yield an product quality model and the optimization algorithm. The "Planner" version will use a nominal set of process information. He can input prices, and propose process operation in terms of setpoints and constraint limits (inside the valid model range). He can then run "what-if" analyses using the model to predict the outcome, or run the optimization function to find the optimal combination of variables while respecting the defined constraints.

The "Operations" version have a model that is running continuously in real-time mode in a process computer. Special functions detects if the measurement quality is $\mathrm{OK}$ and if the process is at steady state. If so, bias adjustments are made to the various models, so in effect a self-calibration takes place. Optimization calculations are then run, and the results are presented to the operators as recommended setpoints or limit values. The economic result of the operation is continuously available, and the system 
allows the operator to perform "what-if" analyses to explore the economic consequences of alternative operation modes. The expected frequency of use is once or twice a shift.

We apply a commercially available sequential quadratic programming (SQP) routine to solve the optimization problem. An optimizer prototype was developed in Matlab, while the final version is in FORTRAN. At the time being, we expect the optimization problem to have approximately $20-25$ independent variables and about 40 process model equations. For the optimization, the economics (objective function) is calculated under the restriction of entire plant mass balance satisfaction in the prediction, while the constraint predictions are based on predicted delta values from the current operating point.

The price of one product can take two stages. Up to a specified flow rate limit the price reflect the more preferable use of the product, while the price above that limit reflects for instance the market price of the blend component when sold as such. In addition, as for the naphtha, the price function also depends on product quality, reflecting for instance that more Cracker naphtha can be utilized for gasoline product blending when the octane number is high. Further flexibility in the product specification is introduced by allowing explicit constraints on product rate and quality.

If the self-calibration functions proves to be robust enough, it would be tempting to close the loop by automatically transferring the recommended setpoints and limit values to the MPC controllers.

\section{Discussion}

Optimization functions differs much in complexity and validity range for the underlying models. This is also reflected in the big variation in cost for such functions. In this case it was proposed to go for a "hybrid" model, combining relations known from theoretical cracker models with empirical regression techniques. The usage of such a model has to be limited to a proven operation range, but that is all that is needed for short-term planning purposes. The possible drawback is that models have to be checked, and probably modified, if the process mode of operation or catalyst properties are changed significantly. So far, the complexity of the described optimization model seems to be a very cost-effective compromise between the big-scale rigorous models and the empirical linear regressions models. The tailoring and specialization inherent in this type of model is a two-edged sword, because there is no external vendor to turn to if things have to be changed. The moral is: keep functions and models so simple that they can be maintained in-house, and keep up the know-how by keeping up similar work.

There are a lot of remaining challenges to discuss and solve along the line of the optimization project:

- The precision and details of the Linear Programming type of model used for long-term planning is less than the specially adapted non-linear models. How do we assure consistency between the outcome of monthly and weekly production planning and the day-to-day plant optimization?

- What prices to use for feeds, products and intermediate streams? Should the on-line optimizer use prices at the moment of production planning, at the production time, or predicted sales price for future contracts?

- How much of the information and decision making currently done by production planners could, or should, be transferred to panel operators? Similar optimisers 
could be implemented for other main processes. All of these would give local optimums. How tightly should such functions be coupled?

- Intermediate product storage is not considered in this optimization problem. How much flexibility is needed to handle unforeseen supply or delivery problems?

These questions are still up for discussion, and there seem not to be a simple, single answer, although we have already indicated that the development of some sort of a price-balancing mechanism on a shorter term than the typical LP-scope will be essential. Some improvements might also be achieved incorporating some of the new process knowledge in the overall refinery LP model which is used for monthly production planning.

Supervisory control and optimization have posed some new challenges of a non-technical nature. Traditional long-term planning, production scheduling, and daily operation in a refinery are separate, disjointed activities, each of them being a defined organizational function with defined boundaries. Transfer of results from one level to the next is by formal, written instructions. The new computerization links these functions closer together, but it also blurs the lines which defines responsibilities.

Optimizer functions really do tell what combination of limits and setpoints will maximize the defined objective function, without knowing whether the objective is correctly defined. Operation engineers can end up being guardians of the physical limits of the process, reflecting all economic responsibility back to the production planner, who put in the price numbers. Then, to complete the picture, the production planner could blame the (programmers of the) optimizer. To avoid this situation, a full understanding of the role, the benefits, and the limitations of the various functions in the hierarchy of control is essential.

The cracker optimization project is in many ways a pilot project in this field. The expected benefits come from the difference in added value running the process traditionally, compared to running with an "optimal" combination of setpoints, calculated based on a self-calibrating process model, real constraints and up-dated prices. An on-line model provides the operators with new information about the economic consequences of any changes made to the operation. Traditionally, operators are trained to care about the physical state of the process, the economic part is left for others. This attitude is challenged nowadays, and an on-line optimization function may facilitate a new "economic awareness" in the control room. So far we have realized that a co-ordinated and unified approach involving all parties is essential, further that a technically well-performing planning tool is just the beginning, and finally that the organizational division of responsibilities and the competence and motivation of the users must match the technical system hierarchy in order to produce the expected results.

\section{Conclusion}

The MPC applications in the Cracker plant are well accepted by the operators, and have added control benefits in the order of magnitude which was expected when initializing the project. Some important factors contributing to this are:

- The IDCOM controller has a well-defined control priority hierarchy, allowing application designs meeting most operational objectives.

- The operations department devoted a full-time person to the project.

- There were internal resources available to perform a substantial monitoring, 
tuning and re-designing effort for almost a year after the applications were up running.

- The applications continuously provide stable control of the process at sets of active operational constraints which change with time.

Doing such a project, one should remember that dynamic plant testing and model identification is critical. The following factors are the more important ones:

- instrumentation performance (valves and actuators)

- that the PRBS is run with appropriate frequency range and constraint variables close to the limits and with handles for the operator to keep controlling the process satisfactorily

- and preferably, use an identification tool which accepts steady-state gains as inputs if other available data can be used to determine those gains

On-line economical optimization is currently being implemented as a function on top of the MPC system, due to the complicated process with a lot of interactions and conflicting economics when each product is considered separately. The product yields and qualities are predicted by models established from a large set of plant data coming from carefully designed experiments. The choice of model structure has to some extent been motivated from theoretical studies. This type of hybrid regression models seems to fit well to the on-line optimization function. In the optimization context it is important to focus on testing and modelling of those responses which, set together to the final system, can allow you to take out $80-90 \%$ of the potential.

There is hardly any technological limitation on the benefits which supervisory control and optimization can add to the process operations. When it comes to organizational aspects, however, there are some precautions to keep in mind:

- Be sure to develop the internal competence level appropriately.

- Keep models and functions so simple that they can be maintained in-house.

- Define and implement properly the organizatorial ownership and responsibility of the new functions.

- Calculate with a lot of internal resources devoted to the task of maintaining the functions routinely, otherwise they would degrade along with changes in process and objectives.

Successful projects in this field really add economical benefits.

\section{REFERENCES}

LJUNGQUIST, D. (1990). Online estimation in nonlinear state-space models with application to catalytic cracking. Dr. Ing. thesis. Report 90-89-W, Division of Engineering Cybernetics, NTH, Trondheim.

StRAND, S. (1991). Dynamic optimization in state-space predictive control schemes. Dr. Ing. thesis. Report 91-11-W, Division of Engineering Cybernetics, NTH, Trondheim. 